\title{
Experimental Study on Dynamic Reactionless Motions with DLR's Humanoid Robot Justin
}

\author{
Thomas Wimböck, Dragomir Nenchev, Alin Albu-Schäffer and Gerd Hirzinger
}

\begin{abstract}
The capabilities of DLR's multi-DOF humanoid robot Justin are extended with the help of a dynamic torque control component for base reaction minimization. Since the mobile base of the robot comprises springs, reactions induced by arm/torso motions lead to vibrations and deteriorate the performance. The control component is derived from the equation of motion of the robot, represented as an underactuated system, and partitioned into a "driven" subsystem (one of the arms), and a "compensating" subsystem (the other arm, with or w/o torso contribution). The control component is then embedded into the existing sophisticated controller structure of Justin, as a feedforward component, with additional control signals from an augmented PD feedback controller. It was possible to obtain satisfactory performance with a very "soft" compensatory subsystem. The experimental results confirmed the potential of this model-based approach for use in a complex multi-DOF system. As far as we know, this is the first time that a dynamic-coupling compensating controller is applied to a real system of such complexity, utilizing thereby a torque control interface.
\end{abstract}

\section{INTRODUCTION}

Motion control for minimizing the reaction at a manipulator base is an important control problem for mobilebase robots, e.g. free-flying space robots, flexible-base space robots, and others.

A class of flexible-base robots, being developed recently, are humanoid-type robots, comprising an anthropomorphic upper body mounted on a mobile base with wheels instead on legs. Such robots have at least two advantages when compared with conventional legged humanoids: (1) they are much more stable since the wheel base of support can be designed appropriately, and (2), they are much more efficient from the point of view of energy consumption. Wheelbase humanoids, on the other hand, have some disadvantage because they can operate only within flat-floor environments. This problem can be alleviated to some extent, though, by including rubber tires and/or spring/dampers for suspension, so that small obstacles can be negotiated, similar to automobiles. Note, however, that the passive elements may introduce significant base deflection, which would deteriorate the accuracy of manipulation. Therefore, such wheel-base robots should be regarded as flexible-base robots, and appropriate methods of control should be applied.

T. Wimböck, A. Albu-Schäffer and G. Hirzinger are with DLR - German Aerospace Center, Institute of Robotics and Mechatronics, P.O. Box 1116, D-82230 Wessling, Germany \{thomas.wimboeck, alin.albu-schaeffer, gerd.hirzinger\}edlr.de

D. Nenchev is with the Department of Mechanical Systems Engineering, Tokyo City University, Tamazutsumi 1-28-1, Setagaya-ku, Tokyo 158-8557, Japan nenchev@ieee. org

D. Nenchev acknowledges the support by Grant-in-Aid for Scientific Research Kiban (B) 20300072 of JSPS.

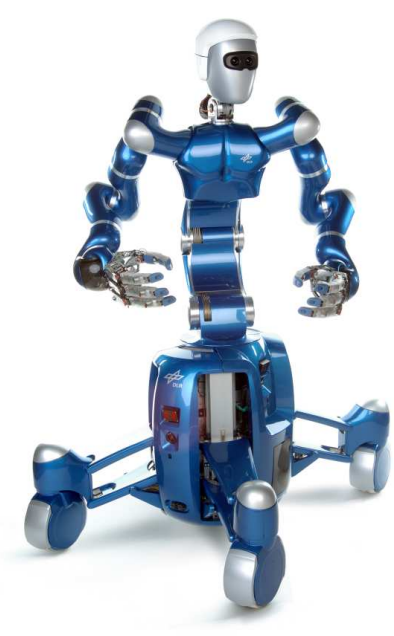

Fig. 1. Justin's photo.

Flexible-base robots represent a challenge from the control point of view. The reason is the dynamic coupling between the motion of the manipulator(s) and that of the flexible base. Vibrations can be induced into the base by a disturbance wrench, imposed via link motion. Base vibration leads in turn to disturbances in the manipulator joint torque, and the system may become unstable at the end. The problem has been tackled in past studies, and various control methods have been proposed. These can be classified into four wide categories:

1) base vibration suppression control methods [1]-[4];

2) design of control inputs that induce minimum vibrations [5];

3) end-point control in the presence of vibrations [6], [7];

4) end-point control for interaction tasks [8].

Appropriate control methods depend very much on the structure of the manipulator, e.g. dual-arm or single-arm, on the presence of kinematic and/or dynamic redundancy and on the availability of sensors for measuring the deflection of the base and/or in the joints. In our previous research, we have proposed controllers for combining vibration suppression with reactionless motion, both for single arm [9] and dual-arm [10] flexible-base manipulators, for simple planar systems with deflection feedback based control.

In this work, we consider DLR's humanoid robot Justin [11] as an experimental platform (see Fig. 1). The robot comprises a torso with two arms, two hands and a head, 
and is mounted on a mobile base with built-in spring and dampers [12]. The two arms are made of two seven-DOF DLR lightweight manipulators DLR-LWR-III, with flexible joints and built-in joint torque sensors. The torso is a fourjoint three-DOF spatial system, the upper joint being driven in a coupled manner via a cable drive. Thus, in addition to the flexibilities in the base and in the joints, there is also flexibility in the cable drive. The controller of the robot consists basically of two closed loops - a fast $(3 \mathrm{kHz})$ inner torque feedback loop, and a passivity-based outer impedance control loop [13]. With this controller, structural vibrations can be suppressed at the individual joint level. The controller does not account for base vibrations.

The main goal of this work is to extend the capability of the existing controller towards base reaction minimization, with the help of a model-based, inertia-coupling feedback control method, similar to that used in previous studies [9], [10]. Note, however, that in these or other similar studies velocity-driven controllers have been addressed. As far as we know, this is the first time that an inertia-coupling controller will be applied to a real system of such complexity, utilizing thereby a torque control interface. We emphasize thereby that we intend to design torque control inputs for the upper body links, that would induce minimum dynamic deflections into the base. For treatment of a similar problem regarding static deflections, the interested reader is referred to [8].

\section{BACKGROUND AND NOTATION}

We will base our derivations on the Reaction Null Space concept developed some time ago for free-flying [14] and flexible-base manipulators [9] in zero gravity environment. Recently, it was shown also that the concept can be applied to humanoid robots (nonzero gravity), for controlling the balance via the reaction imposed on the foot [15].

The equation of motion of a rigid-body multilink robot system comprising $n$-joints, mounted on a flexible base with $k$ flexible coordinates, can be written in the following form:

$$
\begin{aligned}
{\left[\begin{array}{cc}
\boldsymbol{H}_{b} & \boldsymbol{H}_{b m} \\
\boldsymbol{H}_{b m}^{T} & \boldsymbol{H}_{m}
\end{array}\right]\left[\begin{array}{c}
\dot{\boldsymbol{\nu}}_{b} \\
\ddot{\boldsymbol{q}}
\end{array}\right]+\left[\begin{array}{c}
\boldsymbol{c}_{b} \\
\boldsymbol{c}_{m}
\end{array}\right]+\left[\begin{array}{c}
\boldsymbol{g}_{b} \\
\boldsymbol{g}_{m}
\end{array}\right] } \\
+\left[\begin{array}{c}
\boldsymbol{D}_{b} \boldsymbol{\nu}_{b} \\
\mathbf{0}
\end{array}\right]+\left[\begin{array}{c}
\boldsymbol{K}_{b} \Delta \boldsymbol{\xi} \\
\mathbf{0}
\end{array}\right]=\left[\begin{array}{l}
\mathbf{0} \\
\boldsymbol{\tau}
\end{array}\right]
\end{aligned}
$$

where $\Delta \boldsymbol{\xi} \in \Re^{k}$ denotes the positional and orientational deflection of the base from its equilibrium, $\nu_{b}$ is the twist (velocity/angular velocity) of the base, $\boldsymbol{q} \in \Re^{n}$ stands for the joint coordinates of the robot links, $\boldsymbol{H}_{b}(\boldsymbol{q}, \Delta \boldsymbol{\xi}), \boldsymbol{D}_{b}$, and $\boldsymbol{K}_{b} \in \Re^{k \times k}$ denote base inertia, damping and stiffness matrices, respectively. $\boldsymbol{H}_{m}(\boldsymbol{q}) \in \Re^{n \times n}$ is the inertia matrix of the upper body and $\boldsymbol{H}_{b m}(\boldsymbol{q}, \Delta \boldsymbol{\xi}) \in \Re^{k \times n}$ denotes the so-called inertia coupling matrix, which plays an important role in the following derivations. Vectors $\boldsymbol{c}_{b}\left(\boldsymbol{q}, \dot{\boldsymbol{q}}, \Delta \boldsymbol{\xi}, \boldsymbol{\nu}_{b}\right)$ and $\boldsymbol{c}_{m}\left(\boldsymbol{q}, \dot{\boldsymbol{q}}, \Delta \boldsymbol{\xi}, \boldsymbol{\nu}_{b}\right)$ include velocity-dependent nonlinear terms, $\boldsymbol{g}_{b}(\Delta \boldsymbol{\xi}, \boldsymbol{q})$ and $\boldsymbol{g}_{m}(\Delta \boldsymbol{\xi}, \boldsymbol{q})$ are the gravity forces on the base and on the links, respectively. The vector $\tau \in \Re^{n}$ is the joint torque produced by the motors. No external forces are acting neither on the base nor on the links.
Note that the above equation of motion does not include link or joint flexibilities. In fact, the experimental robot we intend to use, Justin, comprises joint flexibilities, as already mentioned. It is possible, though, to treat this robot as a rigid-joint system, under the two-time scale notation and the singular perturbation approach [13].

For the multi-DOF case under consideration, we can assume that $n \gg k$, which means there are abundant active redundant DOFs. This redundancy can be used to minimize the wrench imposed on the flexible base coordinates via the upper body motion. Assuming a motionless base $\left(\boldsymbol{\nu}_{b}=\mathbf{0}\right)$ at static equilibrium $\left(\boldsymbol{g}_{b}=-\boldsymbol{K}_{b} \Delta \boldsymbol{\xi}\right)$, from the upper part of the last equation, we have:

$$
\ddot{\boldsymbol{q}}=-\boldsymbol{H}_{b m}^{+} \boldsymbol{c}_{b}+\left(\boldsymbol{U}-\boldsymbol{H}_{b m}^{+} \boldsymbol{H}_{b m}\right) \boldsymbol{\zeta}
$$

where $\boldsymbol{H}_{b m}^{+} \in \Re^{n \times k}$ denotes the Moore-Penrose generalized inverse of the inertia coupling matrix, $U$ is the unit matrix of proper dimension, and $\zeta$ is an arbitrary vector having the dimension of joint acceleration. This vector is projected via $\boldsymbol{U}-\boldsymbol{H}_{b m}^{+} \boldsymbol{H}_{b m}$ onto the kernel of the inertia coupling matrix. We refer to this kernel as the Reaction Null Space [9].

It should be apparent that the set of joint accelerations, obtained from the above equation, do not contribute to dynamic forces at the base. By inserting these joint accelerations into the lower part of the equation of motion (1), we obtain the respective set of joint torque:

$$
\boldsymbol{\tau}=\boldsymbol{c}_{m}+\boldsymbol{g}_{m}-\boldsymbol{H}_{m} \boldsymbol{H}_{b m}^{+} \boldsymbol{c}_{b}+\boldsymbol{H}_{m}\left(\boldsymbol{U}-\boldsymbol{H}_{b m}^{+} \boldsymbol{H}_{b m}\right) \boldsymbol{\zeta} .
$$

Based on this general form of the dynamics, in the following section it will be shown how to present the dynamical model of a multi-limb robot system, in a form suitable for reaction minimization.

\section{Modeling AND COMPENSATION SCEnARIOS FOR THE TWO-ARM Robot SySTEM}

The model under consideration has a tree-like structure, comprising three branches - the torso, the right and the left arm. The end-link of the torso is connected to the flexible base (see Fig. 2).

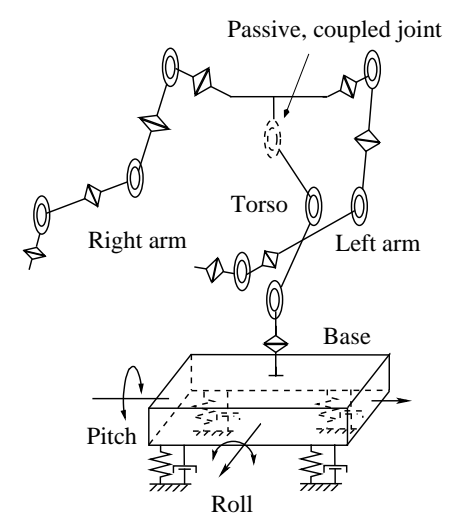

Fig. 2. Model of a humanoid two-arm system on a flexible base. 
The system equation of motion is written as follows:

$$
\begin{gathered}
{\left[\begin{array}{cccc}
\boldsymbol{H}_{b b} & \boldsymbol{H}_{b r} & \boldsymbol{H}_{b t} & \boldsymbol{H}_{b l} \\
\boldsymbol{H}_{b r}^{T} & \boldsymbol{H}_{r r} & \boldsymbol{H}_{r t} & \boldsymbol{H}_{r l} \\
\boldsymbol{H}_{b t}^{T} & \boldsymbol{H}_{r t}^{T} & \boldsymbol{H}_{t t} & \boldsymbol{H}_{t l} \\
\boldsymbol{H}_{b l}^{T} & \boldsymbol{H}_{r l}^{T} & \boldsymbol{H}_{t l}^{T} & \boldsymbol{H}_{l l}
\end{array}\right]\left[\begin{array}{c}
\dot{\boldsymbol{\nu}}_{b} \\
\ddot{\boldsymbol{q}}_{r} \\
\ddot{\boldsymbol{q}}_{t} \\
\ddot{\boldsymbol{q}}_{l}
\end{array}\right]+\left[\begin{array}{c}
\boldsymbol{c}_{b} \\
\boldsymbol{c}_{r} \\
\boldsymbol{c}_{t} \\
\boldsymbol{c}_{l}
\end{array}\right]+\left[\begin{array}{c}
\boldsymbol{g}_{b} \\
\boldsymbol{g}_{r} \\
\boldsymbol{g}_{t} \\
\boldsymbol{g}_{l}
\end{array}\right]} \\
+\left[\begin{array}{c}
\boldsymbol{D}_{b} \boldsymbol{\nu}_{b} \\
\mathbf{0} \\
\mathbf{0} \\
\mathbf{0}
\end{array}\right]+\left[\begin{array}{c}
\boldsymbol{K}_{b} \Delta \boldsymbol{\xi} \\
\mathbf{0} \\
\mathbf{0} \\
\mathbf{0}
\end{array}\right]=\left[\begin{array}{c}
\mathbf{0} \\
\boldsymbol{\tau}_{r} \\
\boldsymbol{\tau}_{t} \\
\boldsymbol{\tau}_{l}
\end{array}\right],
\end{gathered}
$$

where subscripts $b, t, r$ and $l$ stand for base, torso, right arm and left arm, respectively. Notations for the vectors and matrices have the same meaning as those in (1). The double subscripts in the inertia matrix signify inertia coupling properties, e.g. $\boldsymbol{H}_{b r}$ is the inertia coupling matrix between base and right arm, $\boldsymbol{H}_{t l}$ is that between torso and left arm, and so on.

One can think of several strategies for compensation control, depending on the task. There is a class of applications that would require just a single-arm motion. Then, one can assign the desired motion for that arm (e.g. the right arm) in the usual way, and use the other arm (the left arm) for compensation, leaving the torso thereby ideally motionless. Another possibility is to use the links of the torso and that of the left arm for compensation. In other cases, it will be more important to assign a desired motion to the torso, and use both arms for compensation. Also, there is a dual-arm motion scenario, when both arms hold an object and compensation is done through arm redundancy (if available) [10], and through the torso. In short, because of the abundant DOF's, there are many combinations, and the envisioned controller should be flexible enough to cover all practically valuable scenarios.

Below we consider two representative cases whereby the right arm is executing a specified task, while compensation is done either by the left arm only, or by the left arm and the torso. In either case, we will use the term "driven arm" for the right arm and "compensating subsystem" for the rest.

Consider first the simplest case, when only the left arm is compensating. Since the torso remains motionless, the rows and columns containing subscript $t$ can be taken out of the equation of motion (4):

$$
\begin{gathered}
{\left[\begin{array}{lll}
\boldsymbol{H}_{b b} & \boldsymbol{H}_{b r} & \boldsymbol{H}_{b c} \\
\boldsymbol{H}_{b r}^{T} & \boldsymbol{H}_{r r} & \boldsymbol{H}_{r c} \\
\boldsymbol{H}_{b c}^{T} & \boldsymbol{H}_{r c}^{T} & \boldsymbol{H}_{c c}
\end{array}\right]\left[\begin{array}{c}
\dot{\boldsymbol{\nu}}_{b} \\
\ddot{\boldsymbol{q}}_{r} \\
\ddot{\boldsymbol{q}}_{c}
\end{array}\right]+\left[\begin{array}{c}
\boldsymbol{c}_{b} \\
\boldsymbol{c}_{r} \\
\boldsymbol{c}_{c}
\end{array}\right]+\left[\begin{array}{c}
\boldsymbol{g}_{b} \\
\boldsymbol{g}_{r} \\
\boldsymbol{g}_{c}
\end{array}\right]} \\
+\left[\begin{array}{c}
\boldsymbol{D}_{b} \boldsymbol{\nu}_{b} \\
\mathbf{0} \\
\mathbf{0}
\end{array}\right]+\left[\begin{array}{c}
\boldsymbol{K}_{b} \Delta \boldsymbol{\xi} \\
\mathbf{0} \\
\mathbf{0}
\end{array}\right]=\left[\begin{array}{c}
\mathbf{0} \\
\boldsymbol{\tau}_{r} \\
\boldsymbol{\tau}_{c}
\end{array}\right],
\end{gathered}
$$

where subscript $c$ stands for "compensating." Under the assumption of reactionless motion and stationary base at static equilibrium, the dynamic base constraint is obtained from the first row of the above equation, as:

$$
\boldsymbol{H}_{b r} \ddot{\boldsymbol{q}}_{r}+\boldsymbol{H}_{b c} \ddot{\boldsymbol{q}}_{c}+\boldsymbol{c}_{b}=\mathbf{0} .
$$

With the help of this constraint, we will eliminate the compensating (left arm) joint acceleration $\ddot{\boldsymbol{q}}_{c}$ from the equation of motion. From the last equation, we have:

$$
\ddot{\boldsymbol{q}}_{c}=-\boldsymbol{H}_{b c}^{+}\left(\boldsymbol{H}_{b r} \ddot{\boldsymbol{q}}_{r}+\boldsymbol{c}_{b}\right)+\left(\boldsymbol{U}-\boldsymbol{H}_{b c}^{+} \boldsymbol{H}_{b c}\right) \boldsymbol{\zeta}_{c},
$$

where the first term on the r.h.s. denotes compensating acceleration (for the reaction from the right arm and for nonlinear coupling), while the second term stands for acceleration from the kernel of the inertia coupling matrix of the left arm. The latter acceleration will not contribute to base disturbance. Henceforth, we will make use just of the compensating acceleration, assuming the arbitrary vector $\boldsymbol{\zeta}_{c}=\mathbf{0}$.

The joint acceleration from the last equation is substituted into the second and third rows of the equation of motion (5). Then, the joint torque of each arm becomes a function of the joint acceleration of the driven arm (the right arm). Thus, the right-arm joint torque is:

$$
\boldsymbol{\tau}_{r}=\tilde{\boldsymbol{H}}_{r r} \ddot{\boldsymbol{q}}_{r}+\tilde{\boldsymbol{c}}_{r}+\boldsymbol{g}_{r},
$$

where $\tilde{\boldsymbol{H}}_{r r} \equiv\left(\boldsymbol{H}_{r r}-\boldsymbol{H}_{r c} \boldsymbol{H}_{b c}^{+} \boldsymbol{H}_{b r}\right)$ and $\tilde{\boldsymbol{c}}_{r} \equiv \boldsymbol{c}_{r}-$ $\boldsymbol{H}_{r c} \boldsymbol{H}_{b c}^{+} \boldsymbol{c}_{b}$ ). The left arm (the compensating arm) joint torque is:

$$
\boldsymbol{\tau}_{c}=\tilde{\boldsymbol{H}}_{r c}^{T} \ddot{\boldsymbol{q}}_{r}+\tilde{\boldsymbol{c}}_{c}+\boldsymbol{g}_{c}
$$

where $\tilde{\boldsymbol{H}}_{r c}^{T} \equiv\left(\boldsymbol{H}_{r c}^{T}-\boldsymbol{H}_{c c} \boldsymbol{H}_{b c}^{+} \boldsymbol{H}_{b r}\right)$ and $\tilde{\boldsymbol{c}}_{c} \equiv \boldsymbol{c}_{c}-$ $\boldsymbol{H}_{c c} \boldsymbol{H}_{b c}^{+} \boldsymbol{c}_{b}$.

Next, consider the case when compensation is done also with the torso, in addition to the left arm. Referring to (4), we introduce the following notation:

$$
\begin{gathered}
\boldsymbol{H}_{c c} \equiv\left[\begin{array}{ll}
\boldsymbol{H}_{t t} & \boldsymbol{H}_{t l} \\
\boldsymbol{H}_{t c}^{T} & \boldsymbol{H}_{l l}
\end{array}\right], \boldsymbol{q}_{c} \equiv\left[\begin{array}{l}
\boldsymbol{q}_{t} \\
\boldsymbol{q}_{l}
\end{array}\right], \boldsymbol{\tau}_{c} \equiv\left[\begin{array}{l}
\boldsymbol{\tau}_{t} \\
\boldsymbol{\tau}_{l}
\end{array}\right], \\
\boldsymbol{c}_{c} \equiv\left[\begin{array}{ll}
\boldsymbol{c}_{t}^{T} & \boldsymbol{c}_{l}^{T}
\end{array}\right]^{T}, \boldsymbol{g}_{c} \equiv\left[\begin{array}{ll}
\boldsymbol{g}_{t}^{T} & \boldsymbol{g}_{l}^{T}
\end{array}\right]^{T}, \\
\boldsymbol{H}_{b c} \equiv\left[\begin{array}{ll}
\boldsymbol{H}_{b t} & \boldsymbol{H}_{b c}
\end{array}\right], \boldsymbol{H}_{r c} \equiv\left[\begin{array}{ll}
\boldsymbol{H}_{r t} & \boldsymbol{H}_{r c}
\end{array}\right] .
\end{gathered}
$$

With this notation, we can represent the original equation of motion (4) again in the reduced form (5).

\section{Control Law Formulation}

We assume that the driven arm (right arm) tracks an arbitrary trajectory $\left(\boldsymbol{q}_{r}^{d}, \dot{\boldsymbol{q}}_{r}^{d}, \ddot{\boldsymbol{q}}_{r}^{d}\right)$, assigned in joint coordinates ${ }^{1}$. During path tracking, a nonzero wrench will be then imposed on the base from the right arm. In order to minimize the total reaction at the base, this wrench will be compensated by a wrench generated by the compensating subsystem.

This strategy will be realized with the help of a torque controller, having the capability to deal with dynamic motions. We employ a model-based approach, whereby the compensating wrench is generated via a desired torque component. This component is obtained, in turn, from the joint acceleration of the compensation subsystem, as given in $(7)$ :

$$
\ddot{\boldsymbol{q}}_{c}^{d}=-\boldsymbol{H}_{b c}^{+}\left(\boldsymbol{H}_{b r} \ddot{\boldsymbol{q}}_{r}^{d}+\boldsymbol{c}_{b}\right) .
$$

In addition to the desired acceleration $\ddot{\boldsymbol{q}}_{c}^{d}$, we assign a desired final state for the compensating subsystem, preferably the stationary one: $\dot{\boldsymbol{q}}_{c}^{d}=\mathbf{0}$ and $\boldsymbol{q}_{c}^{d}=$ const. One

\footnotetext{
${ }^{1}$ Superscript $(0)^{d}$ denotes a desired value.
} 
reasonable choice for the desired configuration $\boldsymbol{q}_{c}^{d}$ is the initial one. Hence, a regulator-type configuration controller with feedforward component will be obtained. Other choices for the behavior of the compensatory subsystem are also possible, e.g. assigning a desired path for its CoM, such that gravity based disturbance wrenches will be compensated, in addition to the dynamic ones envisioned here.

Further on, we set the desired motion of the base to be stationary, for achieving reactionless motion, i.e. $\dot{\nu}_{b}^{d}, \boldsymbol{\nu}_{b}^{d}$ and $\Delta \boldsymbol{\xi}^{d}$ all are zeros.

A reasonable choice for a joint-space dynamic trajectory tracking controller is the augmented PD controller [16]. The joint damping and stiffness torque components of this controller can then be matched with those of the original controller of Justin, used for adjusting the joint impedance.

The augmented PD controller is written as:

$$
\boldsymbol{\tau}^{d}=\boldsymbol{H}(\boldsymbol{q}) \ddot{\boldsymbol{q}}^{d}+\boldsymbol{c}\left(\boldsymbol{q}, \dot{\boldsymbol{q}}, \dot{\boldsymbol{q}}^{d}\right)+\boldsymbol{g}(\boldsymbol{q})-\boldsymbol{K}_{d} \dot{\boldsymbol{e}}-\boldsymbol{K}_{p} \boldsymbol{e},
$$

where

$$
\boldsymbol{q}=\left[\begin{array}{c}
\Delta \boldsymbol{\xi} \\
\boldsymbol{q}_{r} \\
\boldsymbol{q}_{c}
\end{array}\right], \boldsymbol{\tau}=\left[\begin{array}{c}
\mathbf{0} \\
\boldsymbol{\tau}_{r} \\
\boldsymbol{\tau}_{c}
\end{array}\right], \boldsymbol{g}=\left[\begin{array}{l}
\boldsymbol{g}_{b} \\
\boldsymbol{g}_{r} \\
\boldsymbol{g}_{c}
\end{array}\right], \boldsymbol{e}=\left[\begin{array}{l}
\boldsymbol{e}_{b} \\
\boldsymbol{e}_{r} \\
\boldsymbol{e}_{c}
\end{array}\right],
$$

the inertia matrix $\boldsymbol{H}$ contains $3 \times 3$ submatrices, as those shown in (5), $\boldsymbol{K}_{p}$ and $\boldsymbol{K}_{d}$ denote positive definite feedback gain matrices, the nonlinear term $\boldsymbol{c}\left(\boldsymbol{q}, \dot{\boldsymbol{q}}, \dot{\boldsymbol{q}}^{d}\right)$ is of the form $\boldsymbol{C}(\boldsymbol{q}, \dot{\boldsymbol{q}}) \dot{\boldsymbol{q}}^{d}[16]$, and the errors are $\boldsymbol{e}_{b}=\Delta \boldsymbol{\xi}, \boldsymbol{e}_{i}=\boldsymbol{q}_{i}-$ $\boldsymbol{q}_{i}^{d}, i \in\{r, c\}$. The closed-loop equation is:

$$
\boldsymbol{H}(\boldsymbol{q}) \ddot{\boldsymbol{e}}+\boldsymbol{C}(\boldsymbol{q}, \dot{\boldsymbol{q}}) \dot{\boldsymbol{e}}+\boldsymbol{K}_{d} \dot{\boldsymbol{e}}+\boldsymbol{K}_{p} \boldsymbol{e}=\mathbf{0} .
$$

It should be noted that, when a regulator-type configuration controller for the compensating subsystem is employed, an additional term should appear in the above equations, stemming from the nonzero desired acceleration (10). This term can be regarded as a disturbance, which will be compensated by the feedback control torque, in addition to other sources of disturbance, e.g. residual joint friction.

The linearizing control joint torque for the right arm is obtained from (11) in the following form:

$$
\boldsymbol{\tau}_{r}^{d}=\tilde{\boldsymbol{H}}_{r r} \ddot{\boldsymbol{q}}_{r}^{d}+\tilde{\boldsymbol{c}}_{r}+\boldsymbol{g}_{r}-\boldsymbol{\Delta}_{d r}\left(\boldsymbol{K}_{d}, \dot{\boldsymbol{e}}\right)-\boldsymbol{\Delta}_{p r}\left(\boldsymbol{K}_{p}, \boldsymbol{e}\right)
$$

and that for the compensating subsystem, as:

$$
\boldsymbol{\tau}_{c}^{d}=\tilde{\boldsymbol{H}}_{r c}^{T} \ddot{\boldsymbol{q}}_{r}^{d}+\tilde{\boldsymbol{c}}_{c}+\boldsymbol{g}_{c}-\boldsymbol{\Delta}_{d c}\left(\boldsymbol{K}_{d}, \dot{\boldsymbol{e}}\right)-\boldsymbol{\Delta}_{p c}\left(\boldsymbol{K}_{p}, \boldsymbol{e}\right) .
$$

The $\Delta$ terms are linear in the errors, and can be computed in a straightforward manner. The ( $\tilde{\circ})$ quantities have the same meaning as in (8) and (9).

It is seen that the two control joint torques are linear functions of the desired joint acceleration of the driven arm. Comparing these control torques with the joint torques (8) and (9), respectively, it becomes apparent that the feedback error $\boldsymbol{\Delta}$ terms will induce some base disturbance. This disturbance will be small, though, as long as the errors remain small. This can be ensured by appropriate feedback gain selection. As noted in [16], gain selection for the augmented PD controller requires some care, especially in the case of trajectory tracking, i.e. for the right arm in our case. We will come back to this problem in the following section, which discusses implementation issues. What should be mentioned here is that, intuitively, the feedback gains for the right arm should have higher values for achieving best trajectory tracking performance, while that for the compensating subsystem should have smaller values, to avoid interference with the compensating, feedforward component, as much as possible.

Another important point is related to the specific feedforward acceleration component of the compensating subsystem, given in (10). Because of this component, the ( $\left.\tilde{o}^{\circ}\right)$ quantities are all functions of the pseudoinverse of the inertia coupling matrix $\boldsymbol{H}_{b c}$. Hence, any rank-deficiency of this matrix should avoided. In other words, well-conditioned inertia coupling is a necessary condition for this controller to work appropriately.

\section{EXPERIMENTAL VERIFICATION WITH JUSTIN}

The kinematic structure of Justin is the one shown in Fig. 2. Justin has two seven-DOF arms, attached to a torso with four joints. The torso has only three DOFs, though, since the motion in the joint closest to the arms, is not independent [11]. Justins's body is mounted on a sophisticated mobile base, with four wheels attached to extendable legs. The legs are connected via four sliding joints, comprising spring/dampers, to the base of the torso. Thus, the torso base has three DOFs for motion in the plane, plus four active DOFs for extending/retracting the legs, in parallel with four spring/dampers [12]. In our experiments, though, the base is modeled just as a passive structure with two angular deflections, denoted as "roll" and "pitch", which contribute to torso base rotation within the frontal and the sagittal plane, respectively (cf. Fig. 2). Altogether, our model has 17 jointDOF and 2 flexible coordinates.

We have integrated the augmented PD motion controller from the previous section into Justin's control structure. As already noted, Justin can be treated as a rigid-joint manipulator under the singular perturbation assumption and the fast inner-loop joint torque feedback controller [13]. Hence, the augmented PD controller (11) is applicable. The PD feedback gains are set according to the requirements of Justin's original controller, such that the damping feedback gain matrix $\boldsymbol{K}_{d}$ is configuration dependent, calculated via the system inertia matrix [13], while the $\mathrm{P}$ feedback gain matrix $\boldsymbol{K}_{p}$ is a constant diagonal matrix. All configurationdependent quantities in the control law (the inertia submatrices and the gravity vectors) are calculated using the measured values of the joint coordinates. The nonlinear terms are represented as vectors $\boldsymbol{c}_{(\circ)}$ and calculated via measured joint angles and desired joint angular velocities, therefore.

We performed three sets of experiments, as follows:

1) compensating subsystem: left arm and torso, with system $\mathrm{P}$ feedback gains as shown in Table I;

2) compensating subsystem: left arm only, with system $P$ feedback gains as shown in Table I; 


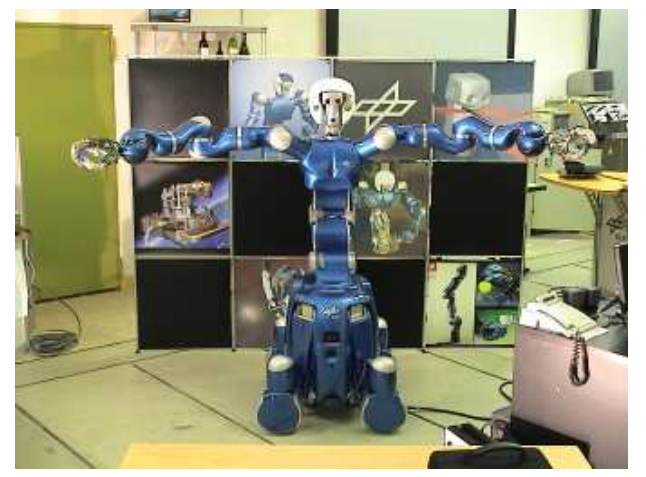

Fig. 3. The initial configuration of Justin during the experiments.

TABLE I

Constant P FEEDBACK GAINS [Nm/rad] $\times 100$

\begin{tabular}{|c|c|c|}
\hline right arm & torso & left arm \\
\hline \hline $7,7,5,5,2,3,3$ & $15,15,15$ & $0.3,0.3,0.3,0.3,0.12,0.12,0.12$ \\
\hline
\end{tabular}

3) same as 2) above, only the P feedback gains of the left arm were decreased by a factor of 10 .

Two experiments were performed for each set, to obtain data for comparing results with and without compensation. The initial configuration of Justin is symmetrical, with both arms almost fully extended along the horizontal (cf. Fig. 3). The desired motion is a rotation in the second joint of the right arm (the driven arm), of 30 deg for about 0.55 $\mathrm{s}$, with third-order spline interpolation. The peak speed achieved thereby is $1.5 \mathrm{rad} / \mathrm{s}$. The resulting, mainly vertical acceleration of the arm CoM and the angular accelerations of the links generate then a disturbance wrench with prevailing torque component around base roll. This wrench is evaluated with the force sensors of the base, integrated into the spring/damper assemblies of the four wheel extension/retraction legs. The desired state of the compensating subsystem equals the initial one.

In all three sets of experiments we obtained almost identical results. Figure 4 shows data from the first set of experiments, as a representative example. The desired joint torque data plots of the right arm are displayed in Fig. 4a. The largest contribution is that of joint 2 , which is the driven joint. The initial and final jumps due to the acceleration feedforward component are clearly seen. After the jumps, the curves are rounded, which is due to the contribution of the relatively high-gain P feedback components. The desired motion is tracked faithfully, as seen also from the joint error norm plots in Fig. 4c.

Next, Fig. 4b shows the desired joint torque plots from the left arm, which has dominant contribution for the compensatory motion. This is especially true for the motion in joint 2 , which should be expected, since the initial configuration is symmetric. The triangular shape of the feedforward component, corresponding to the cubic interpolation, can be clearly recognized. It looks undistorted, because of the relatively low
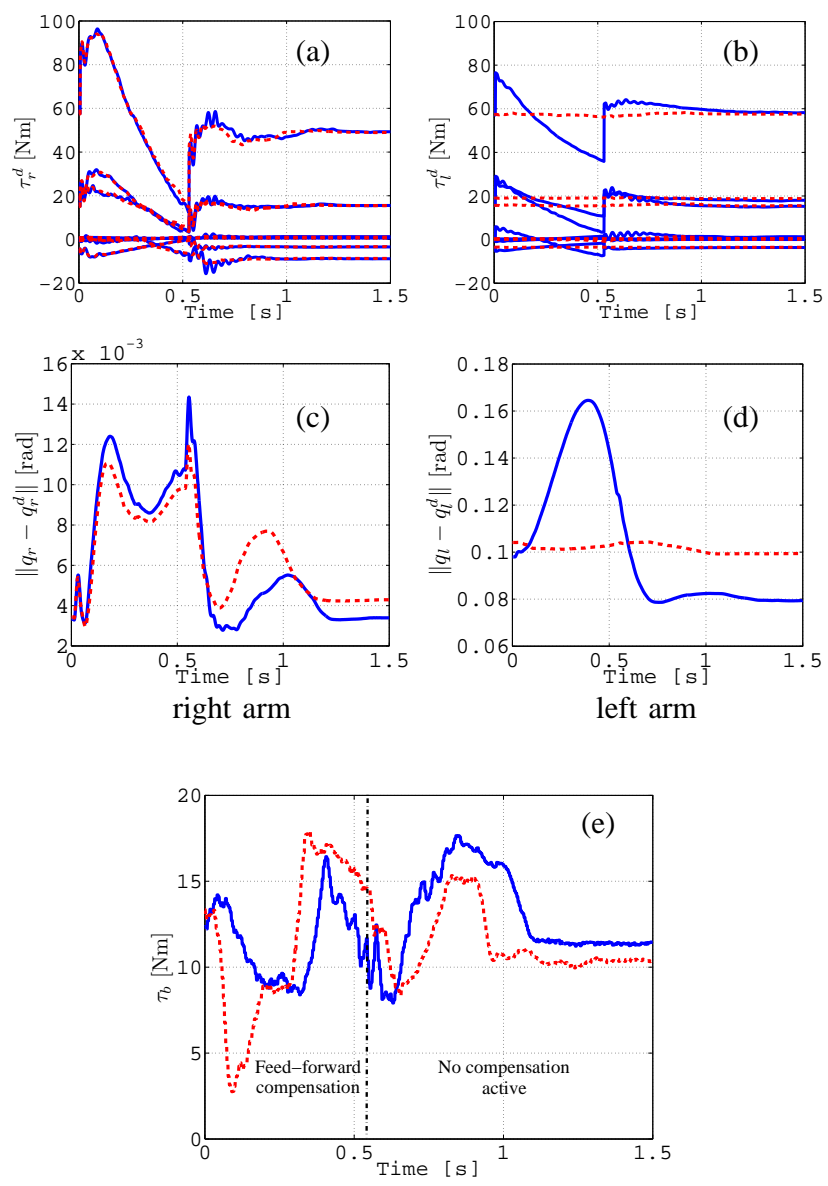

Fig. 4. Compensation with left arm and torso (a), (b): desired joint torque; (c), (d): joint error norm; (e): base roll disturbance torque; red (dashed) without compensation, blue (solid) - with compensation.

$P$ feedback gains. It can be also seen that the rest of the joints contribute to the feedforward compensating motion as well. This is in contrast with the right arm motion, where the rest of the joints contribute to feedback components only. From the respective error plot - the solid blue graph in Fig. 4d - it is seen that the arm configuration changes thereby only slightly.

Figure $4 \mathrm{e}$ shows the plots of the disturbance moments $\tau_{b}$, measured at the flexible base. First, we focus on the acceleration phase (the first $0.35 \mathrm{~s}$ ). Without compensation (red dashed graph), a large variation of $\tau_{b}$ is observed, which is due to the induced reaction. The variation is significantly smaller, in the case when compensation is applied (the solid blue graph). This is actually the main result of this study. Next, we consider the deceleration phase (between 0.35$0.7 \mathrm{~s}$ ). It is seen that the disturbance is not canceled that efficiently, which can be attributed to the contribution of the feedback components. Finally, focusing on the stabilizing phase (after $0.7 \mathrm{~s}$ ), it becomes apparent that the compensating arm introduces some additional disturbance because of larger settling time due to the low $\mathrm{P}$ gains.

With the help of Fig. 5, we can go into further detail about the role of the $\mathrm{P}$ gains of the compensating subsystem. 

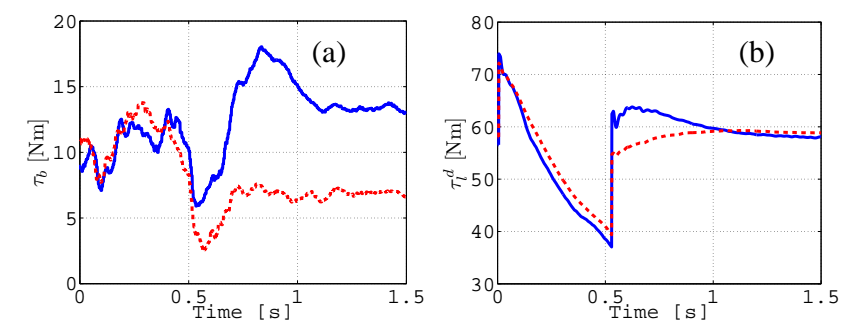

Fig. 5. Performance comparison with low/high $P$ feedback gains. (a): base roll disturbance torque; (b): desired joint torque for left arm joint 2; red (dashed) - low gain, blue (solid) — high gain.

The data shown compares the performance of left arm compensation with high gains (experiment set 2)), with that of low gain compensation (experiment set 3)). From the base disturbance plots in Fig. 5a it is seen that high gain response leads to a larger disturbance during the settling phase, while that of low gain - to larger disturbance during the deceleration phase. From the desired joint torque plots for left arm joint 2 in Fig. $5 b$, it can be seen that the high gain case leads to underdamped response while that of low gain - to overdamped. This demonstrates that careful gain selection plays a crucial role for best performance, indeed.

\section{CONCLUSIONS}

We developed a model-based control method for dynamic reaction minimization with a multi-DOF humanoid-type robot mounted on a flexible base. Our approach designates a particular subsystem of the robot, e.g. one of the arms, as the driven subsystem, performing a given task in the conventional way, while the rest of the links, constituting the compensating subsystem, are minimizing the reaction via a feedforward term. In the same time, a PD feedback regulator ensures that the configuration of the compensating subsystem changes only slightly, and returns to the initial state after task completion.

The control law was implemented with the experimental two-arm robot Justin, developed at DLR. Although there are numerous past studies on reaction minimization control for flexible base robots, and for other types of mobile base robots as well, this is the first time reaction minimization has been practically achieved with such a complex system, and in addition to that, using a torque control interface. Preliminary experiments have shown that the base reaction wrench can be significantly reduced during the acceleration phase of the motion. With this reaction minimization feature, not only the orientation accuracy of the system is increased, but the motion looks also more natural. Indeed, humans use also similar counterbalancing techniques for fast motions. These effects can be clearly observed, also in the video clip accompanying this paper.

Improvements can be made in the following directions. First, because of the model-based approach, parameter accuracy is quite important. In the experiments, the parameters of Justin's arms were fairly well known, for the base, however, only approximate parameters were used. Also, the experiments were conducted with hands and head attached, but their configuration was not accounted for in the model. Second, it was shown that the $\mathrm{P}$ feedback error terms contributed some uncompensated reactions, especially during the deceleration and the settling phase.

Further improvements should be possible by careful $\mathrm{P}$ feedback gain selection for the compensating subsystem. In addition, the error terms can be decomposed via the Reaction Null Space to extract a reactionless component and a minimum norm component, which will help to minimize further the disturbance. Third, the contribution of other unmodeled dynamic effects, mainly nonlinear and velocity-dependent, has to be evaluated as well. Finally, the contribution of residual joint friction, not fully compensated by the joint torque controller, should be examined as well.

\section{REFERENCES}

[1] S. H. Lee and W. J. Book, "Robot vibration control using inertial damping forces," in Proc. of the Eight CISM-IFToMM Symp. RoManSy 8, Cracow, Poland, 1990, pp. 252-259.

[2] M. A. Torres, S. Dubowsky and A. C. Pisoni, "Vibration control of deployment structures' long-reach manipulators: the P-PED method," in Proc. 1996 IEEE Int. Conf. on Robotics and Automation, Minneapolis, Minnesota, April 1996, pp. 2498-2504.

[3] J. Y. Lew and D. J. Trudnowski, "Vibration control of a micro/macro manipulator system," IEEE Control Systems Magazine, Vol. 16, No. 1, Feb. 1996, pp. 26-31.

[4] I. Sharf, "Active damping of a large flexible manipulator with a shortreach robot," Trans. ASME, J. of Dynamic Systems, Measurement and Control, Vol. 118, pp. 704-713, Dec. 1996.

[5] D. W. Cannon et al., "Experimental study on micro/macro manipulator vibration control," in Proc. IEEE Int. Conf. Robotics and Automation, Minneapolis, Minnesota, 1996, pp. 2549-2554.

[6] R. H. Cannon, Jr. and E. Schmitz," Initial experiments on the endpoint control of a flexible one-link robot," Int. J. Rob. Res., Vol. 3, No.3, pp. 62-75, 1984.

[7] C. Mavroidis, S. Dubowsky and V. Raju, "End-point control of long reach manipulator systems," in Proc. Ninth World Congress of IFToMM, Milano, Italy, 1995, pp. 1740-1744.

[8] Ch. Ott, A. Albu-Schäffer, and G. Hirzinger, "A Cartesian Compliance Controller for a Manipulator Mounted on a Flexible Structure," Proc. 2006 IEEE/RSJ Int. Conf. on Intelligent Robots and Systems, Oct. 9-15, 2006, Beijing, China, pp. 4502-4508.

[9] D. N. Nenchev et al., "Reaction Null-Space control of flexible structure mounted manipulator systems," IEEE Tr. on Robotics and Automation, Vol. 15, No. 6, pp. 1011-1023, December 1999.

[10] A. Gouo et al., "Motion control of dual-arm long-reach manipulators," Advanced Robotics, Vol. 13, No. 6, pp. 617-632, 2000.

[11] Ch. Ott et. al., "A Humanoid Two-Arm System for Dexterous Manipulation,” Proc. 2006 IEEE-RAS Int. Conf. on Humanoid Robots, Dec. 4-6, 2006, Genova, Italy, pp. 276-283.

[12] M. Fuchs, P. Robuffo Giordano, Ch. Borst, A. Baumann, E. Kraemer, J. Langwald, R. Gruber, N. Seitz, G. Plank, K. Kunze, R. Burger, F. Schmidt, T. Wimboeck and G. Hirzinger, "Justin's Mobile Platform A Workspace Extension for Two-Handed Manipulation," accepted for presentation at ICRA 09.

[13] Ch. Ott. Cartesian Impedance Control of Redundant and Flexible-Joint Robots. Springer Tracts in Advanced Robotics, Vol. 49, 2008.

[14] D. N. Nenchev, K. Yoshida and Y. Umetani, "Introduction of redundant arms for manipulation in space," IEEE Int. Workshop on Intelligent Robots and Systems, Tokyo, Japan, 1988, pp. 679-684.

[15] D. N. Nenchev and A. Nishio, "Ankle and Hip Strategies for Balance Recovery of a Biped Subjected to an Impact," Robotica, Vol. 26, No. 5, pp. 643-653, 2008.

[16] R. M. Murray, Z. Li, S. S. Sastry, "A Mathematical Introduction to Robotic Manipulation," CRC Press, 1994 (pp. 194-195). 